PROCEEDINGS OF THE

AMERICAN MATHEMATICAL SOCIETY

Volume 133, Number 4, Pages 953-964

S 0002-9939(04)07794-9

Article electronically published on November 19, 2004

\title{
PURELY PERIODIC $\beta$-EXPANSIONS WITH PISOT UNIT BASE
}

\author{
SHUNJI ITO AND HUI RAO
}

(Communicated by David E. Rohrlich)

\begin{abstract}
Let $\beta>1$ be a Pisot unit. A family of sets $\left\{X_{i}\right\}_{1 \leq i \leq q}$ defined by a $\beta$-numeration system has been extensively studied as an atomic surface or Rauzy fractal. For the purpose of constructing a Markov partition, a domain $\hat{X}=\bigcup_{i=1}^{q} \hat{X}_{i}$ constructed by an atomic surface has appeared in several papers. In this paper we show that the domain $\hat{X}$ completely characterizes the set of purely periodic $\beta$-expansions.
\end{abstract}

\section{INTRODUCTION}

1.1. Purely periodic $\beta$-expansion. Let $\beta>1$ be a real number. The $\beta$-expansion of a real number $x \in[0,1]$ is defined as the sequence $\left(x_{i}\right)_{i \geq 1}$ with values in $\{0,1, \ldots,[\beta]\}$ produced by the $\beta$-transformation $T_{\beta}: x \mapsto \beta x(\bmod 1)$ as follows:

$$
\forall i \geq 1, x_{i}=\left\lfloor\beta T_{\beta}^{(i-1)} x\right\rfloor, \text { and thus } x=\sum_{i=1}^{\infty} x_{i} \beta^{-i}=0 . x_{1} \ldots x_{n} \ldots
$$

An expansion is finite if $\left(x_{i}\right)_{i \geq 1}$ is eventually 0 . A $\beta$-expansion is periodic if there exists $p \geq 1$ and $M \geq 1$ such that $x_{k}=x_{k+p}$ holds for all $k \geq M$; if $x_{k}=x_{k+p}$ holds for all $k \geq 1$, then it is purely periodic. We denote by $\operatorname{Per}(\beta)$ the numbers in $[0,1)$ with periodic $\beta$-expansions and by $\operatorname{Pur}(\beta)$ the numbers in $[0,1)$ with purely periodic $\beta$-expansions.

Let $\mathbb{Q}(\beta)$ be the smallest fields containing $\mathbb{Q}$ and $\beta$. An easy argument shows that $\operatorname{Per}(\beta) \subseteq \mathbb{Q}(\beta) \cap[0,1)$ for every real number $\beta>1$. K. Schmidt 17 showed that if $\beta$ is a Pisot number (an algebraic integer whose conjugates have modulus $<1$ ), then $\operatorname{Per}(\beta)=\mathbb{Q}(\beta) \cap[0,1)$. The purely periodic $\beta$-expansions are also discussed in [17.

Property 1 (Schmidt [17). Suppose that $\beta$ satisfies $\beta^{2}=n \beta+1$ for some integer $n \geq 1$. Then every $x \in \mathbb{Q} \cap[0,1)$ has purely periodic $\beta$-expansion.

A number is called a Pisot unit if it is a Pisot number as well as an algebraic unit. The purpose of this paper is to characterize the set $\operatorname{Pur}(\beta)$ when $\beta$ is a Pisot unit. To state our result, we need some preparations.

Received by the editors May 28, 2003.

2000 Mathematics Subject Classification. Primary 11R06; Secondary 37B50.

Key words and phrases. Pisot number, $\beta$-expansion, atomic surface.

The second author was supported by the Japanese Science Promotion Society (JSPS).

(C)2004 American Mathematical Society 
1.2. Automaton of the $\beta$-numeration system. Let $d_{\beta}(1)=\left(b_{i}\right)_{i \geq 1}$ denote the $\beta$-expansion of 1 . Let $d_{\beta}^{*}(1)=d_{\beta}(1)$ if $d_{\beta}(1)$ is infinite, and let $d_{\beta}^{*}(1)=$ $\left(t_{1} \ldots t_{m-1}\left(t_{m}-1\right)\right)^{\infty}$ if $d_{\beta}(1)=t_{1} \ldots t_{m-1} t_{m}$ is finite (with $\left.t_{m} \neq 0\right)$. A sequence is admissible if and only if starting from any place in the sequence, the right side truncation is lexicographically strictly less than $d_{\beta}^{*}(1)$. If the right side truncations are less than or equal to $d_{\beta}^{*}(1)$, then the sequence is called weakly admissible.

When $d_{\beta}^{*}(1)$ is eventually periodic (this holds for any Pisot number), the set of weakly admissible $\beta$-representations can be recognized by the following automaton $M_{\beta}$. Assume that

$$
d_{\beta}^{*}(1)=\left(b_{i}\right)_{i \geq 1}=b_{1} \ldots b_{q-p} \overline{b_{q-p+1} \ldots b_{q}}
$$

so $b_{k+p}=b_{k}$ for all $k>q-p$. Let $r_{k}=b_{k} b_{k+1} \cdots, k \geq 1$. (We denote by $\bar{r}_{k}=0 . b_{k} b_{k+1} \ldots$ the corresponding real number.) Then $\left\{r_{k}\right\}_{k \geq 1}$ is a finite set and actually it is $\left\{r_{1}, \cdots, r_{q}\right\}$. Let the states of $M_{\beta}$ be the integers $1, \cdots, q$ together with a fail state $F$. The initial state is 1 . From a state $j<q, b_{j}$ leads to state $j+1$, while the arrows with labels less than $b_{j}$ lead to state 1 , and all arrows whose labels are greater than $b_{j}$ fail. From state $q, b_{q}$ leads to state $q-p+1$, while all arrows with lower labels lead back to 1 and all arrows with higher labels fail (cf. for example [13, 14, 20]).

1.3. Canonical embedding. We denote by $\mathbb{Z}(\beta)$ the smallest ring containing $\mathbb{Z}$ and $\beta$. From now on we assume that $\beta>1$ is a Pisot unit with minimal polynomial $x^{d}=a_{d-1} x^{d-1}+\cdots+a_{1} x+a_{0}$, where $a_{0}= \pm 1$. Let

$$
A_{\beta}=\left(\begin{array}{cccl}
0 & \cdots & 0 & a_{0} \\
1 & & 0 & a_{1} \\
& \ddots & & \vdots \\
0 & & 1 & a_{d-1}
\end{array}\right)
$$

be the companion matrix of $\beta$. Since $\left\{1, \beta, \cdots, \beta^{d-1}\right\}$ is a basis of the field $\mathbb{Q}(\beta)$, we define a mapping $\phi: \mathbb{Q}(\beta) \mapsto \mathbb{Q}^{d}$ by

$$
\phi\left(z_{1}+z_{2} \beta+\cdots+z_{d} \beta^{d-1}\right)=\left(z_{1}, z_{2}, \cdots, z_{d}\right)^{T},
$$

where $v^{T}$ is the transpose of a vector $v$. Clearly for $x \in \mathbb{Q}(\beta)$, it follows that

$$
\phi(\beta x)=A_{\beta} \phi(x) .
$$

We consider $A_{\beta}$ as a linear transformation on $\mathbb{R}^{d}$. Each real root of the minimal polynomial of $\beta$ is an eigenvalue for this transformation, with a 1-dimensional eigenspace, and for each pair of complex conjugate roots there is a 2-dimensional invariant subspace. Denote by $V$ the eigenspace of $\beta$. Then $V$ is the expanding eigenspace and the other eigenspaces are contractive. Let $P$ be the direct sum of all these contractive eigenspaces. Then $\mathbb{R}^{d}=V \oplus P, \operatorname{dim} P=d-1$ and $\operatorname{dim} V=1$. According to this direct sum we define two natural projections $\pi: \mathbb{R}^{d} \mapsto P$ and $\pi^{\prime}: \mathbb{R}^{d} \mapsto V$. It is easy to see that

$$
A_{\beta} \circ \pi=\pi \circ A_{\beta}, \quad A_{\beta} \circ \pi^{\prime}=\pi^{\prime} \circ A_{\beta} .
$$


1.4. Construction of Markov partition. Let $w_{n} \ldots w_{1} w_{0}$ be an admissible sequence. We say $w_{n} \ldots w_{1} w_{0}$ stops at state $i$ if there is a path with label $w_{n} \ldots w_{1} w_{0}$ which starts from state 1 and ends at state $i$. Let

$$
\begin{aligned}
Y & =\left\{\sum_{i=0}^{n} w_{i} \beta^{i}: w_{n} \ldots w_{1} w_{0} \text { is admissible, } n \geq 0\right\}, \\
Y_{i} & =\left\{\sum_{i=0}^{n} w_{i} \beta^{i}: w_{n} \ldots w_{1} w_{0} \text { is admissible and stops at state } i, n \geq 0\right\} .
\end{aligned}
$$

Clearly $\left\{Y_{1}, \ldots, Y_{q}\right\}$ is a partition of $Y$. Define

$$
X=\overline{\pi \circ \phi(Y)} \text { and } X_{i}=\overline{\pi \circ \phi\left(Y_{i}\right)}, 1 \leq i \leq q .
$$

Then $X=\bigcup_{i=1}^{q} X_{i}$ is a subset of the contractive subspace $P$ and is called the atomic surface by [11 8]. (It is called a Rauzy fractal by [6, 7, 19] and is called a central tile by $[20,3,4]$.) Denote by $e_{1}, \ldots, e_{d}$ the canonical basis of $\mathbb{R}^{d}$, and let

$$
I_{i}=\left\{\theta \pi^{\prime}\left(e_{1}\right): 0 \leq \theta \leq r_{i}\right\}, \quad 1 \leq i \leq q,
$$

be subsets of $V$. Then

$$
\hat{X}_{i}=-X_{i}+I_{i}:=\left\{-x+y: x \in X_{i}, y \in I_{i}\right\}, \quad 1 \leq i \leq q,
$$

are $d$-dimensional tubes. Taking their union, we get

$$
\hat{X}:=\bigcup_{i=1}^{q} \hat{X}_{i}
$$

(See Figure 1.1.) This construction has appeared in several papers (cf. [15, 18, 11. 8]), where its purpose is to construct Markov partitions for the group automorphism $A_{\beta}$. However, in this paper, we will show that $\hat{X}$ completely characterizes the set of purely periodic $\beta$-expansions.

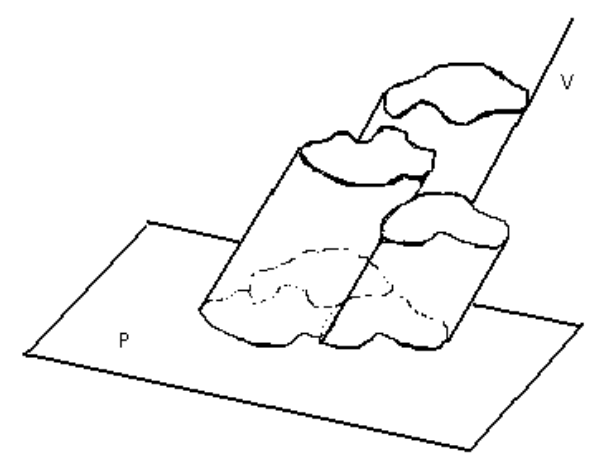

FIGURE 1.1. 
1.5. Main result. Our purpose is to study $\operatorname{Pur}(\beta)$, the set of purely periodic $\beta$ representations which are admissible. However, if we consider $\operatorname{Pur}^{\prime}(\beta)$, the set of purely periodic $\beta$-representations which are weakly admissible, it is slightly different from $\operatorname{Pur}(\beta)$. A purely periodic sequence is weakly admissible but not admissible if and only if it is a shift of the sequence $d_{\beta}^{*}(1)$ and $d_{\beta}^{*}(1)$ is purely periodic. Notice that $d_{\beta}^{*}(1)$ is purely periodic if and only if $d_{\beta}(1)$ is finite. So

$$
\operatorname{Pur}^{\prime}(\beta)= \begin{cases}\operatorname{Pur}(\beta), & \text { when } d_{\beta}(1) \text { is not finite, } \\ \operatorname{Pur}(\beta) \cup\left\{r_{1}, \cdots, r_{q}\right\}, & \text { when } d_{\beta}(1) \text { is finite. }\end{cases}
$$

Since $\operatorname{Pur}(\beta)$ and $\operatorname{Pur}^{\prime}(\beta)$ have very small differences, so in the following we will study $\operatorname{Pur}^{\prime}(\beta)$ instead of $\operatorname{Pur}(\beta)$.

Main Theorem. Let $\beta>1$ be a Pisot unit. Then $x \in \operatorname{Pur}^{\prime}(\beta)$ if and only if $x \in \mathbb{Q}(\beta) \cap[0,1)$ and $\phi(x) \in \hat{X}$.

This theorem has been proved when $\beta$ is of degree 2 by Hara and Ito [10, and proved by Sano and Ito 12 when $\beta$ is a Pisot unit with the minimal polynomial $x^{d}=a_{d-1} x^{d-1}+\cdots+a_{1} x+a_{0}$ where $a_{d-1} \geq a_{d-2} \geq \cdots \geq a_{1} \geq a_{0}=1$. In the present paper, we use a simpler argument and obtain a complete result.

\section{Algebraic version of the Main Theorem}

A two-sided sequence $\left\{a_{i}\right\}_{i \in \mathbb{Z}}$ is (weakly) admissible provided each left truncation $\left\{a_{i}\right\}_{i \geq M}$ is (weakly) admissible. Let $(\Omega, \sigma)$ denote the two-sided symbolic dynamical system associated with $\beta$, where $\Omega$ is the set of admissible two-sided sequences and $\sigma$ the shift operator. We write a two-sided sequence in the form $(w, u)$, where $w=\ldots w_{2} w_{1} w_{0}$ is a backward sequence and $u=u_{1} u_{2} \ldots$ is a forward sequence.

2.1. First realization of $(\Omega, \sigma)$. Let $\beta$ be a Pisot unit of degree $d$. Denote by

$$
\beta=\beta^{(1)}, \beta^{(2)}, \ldots, \beta^{(d)}
$$

the algebraic conjugates of $\beta$. We arrange the sequence $\beta^{(1)}, \beta^{(2)}, \ldots, \beta^{(d)}$ in such an order: the real roots are ahead of the complex roots, and for a complex root $\beta^{(j)}$ we put its complex conjugate $\overline{\beta^{(j)}}$ next to it to make a pair. Suppose $\beta^{(1)}, \ldots, \beta^{(r)}$ are real roots and the others are complex roots. For a backward sequence $w$, we define

$$
\rho(w):=\left(-\sum_{i=0}^{\infty} w_{i}\left(\beta^{(2)}\right)^{i}, \ldots,-\sum_{i=0}^{\infty} w_{i}\left(\beta^{(d)}\right)^{i}\right) .
$$

Abusing the notation a little, we define a projection $\rho: \Omega \mapsto \mathbb{R}^{r} \times \mathbb{C}^{d-r}$ as

$$
\rho(w, u):=\left(\sum_{i=1}^{\infty} u_{i} \beta^{-i}, \rho(w)\right) .
$$

Let $K:=\{\rho(w, u):(w, u) \in \Omega\}$ be the projection of $\Omega$. Clearly $K$ is a bounded subset of $[0,1) \times\left(\mathbb{R}^{r-1} \times \mathbb{C}^{d-r}\right)$. We write a point of $K$ as $(x, y)$, where $x \in[0,1)$ and $y \in \mathbb{R}^{r-1} \times \mathbb{C}^{d-r}$. The last ingredient we need is a mapping $S: K \mapsto K$ defined by

$$
S\left(\begin{array}{l}
x \\
y
\end{array}\right)=I_{\beta}\left(\begin{array}{l}
x \\
y
\end{array}\right)-\lfloor\beta x\rfloor \cdot \mathbf{1},
$$


where 1 denotes the vector $(1,1, \ldots, 1)^{T}$ and $I_{\beta}$ is the diagonal matrix

$$
I_{\beta}=\left(\begin{array}{ccc}
\beta^{(1)} & & 0 \\
& \ddots & \\
0 & & \beta^{(d)}
\end{array}\right)
$$

Proposition 2.1. $(K, S)$ is a realization of $(\Omega, \sigma)$. Precisely, (i) $S \circ \rho=\rho \circ \sigma$, (ii) $S(K)=K$, that is,

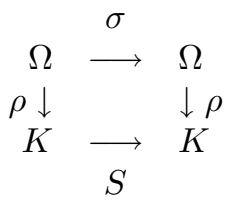

Proof. (i) By direct calculation.

(ii) $S(K)=S \circ \rho(\Omega)=\rho \circ \sigma(\Omega)=\rho(\Omega)=K$.

2.2. Characterization of $\operatorname{Pur}(\beta)$. For $x=c_{0}+c_{1} \beta+\cdots+c_{d-1} \beta^{d-1}$ with $c_{j} \in \mathbb{Q}$, the $j$-th conjugate of $x$ in the field $\mathbb{Q}(\beta)$ is defined by

$$
x^{(j)}=c_{0}+c_{1} \beta^{(j)}+\cdots+c_{d-1}\left(\beta^{(j)}\right)^{d-1} .
$$

We define $x^{\prime}$, the (total) conjugate of $x$, to be $x^{\prime}=\left(x^{(2)}, \cdots, x^{(d)}\right)$. Particularly, $\beta^{\prime}=\left(\beta^{(2)}, \cdots, \beta^{(d)}\right)$. Clearly

$$
\left(\begin{array}{c}
\beta x \\
(\beta x)^{\prime}
\end{array}\right)=I_{\beta}\left(\begin{array}{l}
x \\
x^{\prime}
\end{array}\right) .
$$

Proposition 2.2 illustrates the relation between $S$ and the $\beta$-transformation $T_{\beta}$.

Proposition 2.2. For any $x \in \mathbb{Q}(\beta) \cap[0,1), S\left(x, x^{\prime}\right)=\left(T_{\beta}(x),\left(T_{\beta}(x)\right)^{\prime}\right)$.

Proof. $S\left(\begin{array}{c}x \\ x^{\prime}\end{array}\right)=I_{\beta}\left(\begin{array}{l}x \\ x^{\prime}\end{array}\right)-\lfloor\beta x\rfloor \cdot \mathbf{1}=\left(\begin{array}{c}\beta^{(1)} x^{(1)}-\lfloor\beta x\rfloor \\ \vdots \\ \beta^{(d)} x^{(d)}-\lfloor\beta x\rfloor\end{array}\right)=\left(\begin{array}{c}T_{\beta}(x) \\ \left(T_{\beta}(x)\right)^{\prime}\end{array}\right)$.

Theorem 2.1. Let $\beta>1$ be a Pisot unit. Then $x \in \operatorname{Pur}(\beta)$ if and only if $x \in$ $\mathbb{Q}(\beta) \cap[0,1)$ and $\left(x, x^{\prime}\right) \in K$.

Proof. (i) Suppose $x=0 . \overline{a_{1} \ldots a_{L}} \in \operatorname{Pur}(\beta)$. Then $x \in \mathbb{Q}(\beta) \cap[0,1)$. Let $w=$ $\ldots \overline{a_{1} \ldots a_{L}}$ and $u=\overline{a_{1} \ldots a_{L}} \ldots$. Clearly $(w, u) \in \Omega$. The first coordinate of $\rho(w, u)$ is

$$
x=0 . \overline{a_{1} \ldots a_{L}}=\frac{a_{1} \beta^{L-1}+\cdots+a_{L-1} \beta+a_{L}}{\beta^{L}-1} .
$$

The $j$-th coordinate of $\rho(w, u)$ is $(j \geq 2)$

$$
\begin{aligned}
& -\left(a_{L}+a_{L-1} \beta^{(j)}+\cdots+a_{1}\left(\beta^{(j)}\right)^{L-1}\right)\left(1+\left(\beta^{(j)}\right)^{L}+\left(\beta^{(j)}\right)^{2 L}+\cdots\right) \\
= & \frac{a_{1}\left(\beta^{(j)}\right)^{L-1}+\cdots+a_{L-1}\left(\beta^{(j)}\right)+a_{L}}{\left(\beta^{(j)}\right)^{L}-1}=x^{(j)} .
\end{aligned}
$$

Thus $\left(x, x^{\prime}\right)=\rho(w, u) \in K$. 
(ii) Now we prove the other direction. Suppose $x \in \mathbb{Q}(\beta) \cap[0,1)$ and $\left(x, x^{\prime}\right) \in K$. Let $b$ be the smallest integer such that $b x \in \mathbb{Z}(\beta)$ and set

$$
\mathcal{R}_{b}:=\left\{\left(x, x^{\prime}\right): x \in b^{-1} \mathbb{Z}(\beta)\right\} \cap K
$$

Then $\mathcal{R}_{b}$ is a finite set because $K$ is bounded.

First we assert that $S\left(\mathcal{R}_{b}\right) \subseteq \mathcal{R}_{b}$. For $\left(x, x^{\prime}\right) \in \mathcal{R}_{b}, S\left(x, x^{\prime}\right)=\left(T_{\beta} x,\left(T_{\beta} x\right)^{\prime}\right)$ by Proposition 2.2. Since $T_{\beta} x=\beta x-\lfloor\beta x\rfloor \in b^{-1} \mathbb{Z}(\beta)$ and $S(K)=K$, we have that $S\left(\mathcal{R}_{b}\right) \subseteq \mathcal{R}_{b}$

Secondly we claim that $S$ is surjective on $\mathcal{R}_{b}$. For $\left(x, x^{\prime}\right) \in \mathcal{R}_{b}$, there exists at least one sequence $(w, u) \in \Omega$ such that $\rho(w, u)=\left(x, x^{\prime}\right)$. Let $w_{0}$ be the first symbol of $w$ and let $y=\beta^{-1}\left(x+w_{0}\right)$. We argue that $\rho \circ \sigma^{-1}(w, u)=\left(y, y^{\prime}\right)$. Write

$$
\rho \circ \sigma^{-1}(w, u)=\rho\left(\ldots w_{2} w_{1} \cdot w_{0} u_{1} u_{2} \ldots\right)=\left(t_{1}, t_{2}, \ldots, t_{d}\right)
$$

Then

$$
t_{1}=\frac{w_{0}}{\beta}+\frac{u_{1}}{\beta^{2}}+\frac{u_{2}}{\beta^{3}}+\cdots=\frac{x+w_{0}}{\beta}=y,
$$

and for $j \geq 2$,

$$
\begin{aligned}
t_{j} & =-w_{1}-\beta^{(j)} w_{2}-\left(\beta^{(j)}\right)^{2} w_{3}-\cdots \\
& =\left(\beta^{(j)}\right)^{-1}\left(\left(-w_{0}-\beta^{(j)} w_{1}-\left(\beta^{(j)}\right)^{2} w_{2}-\cdots\right)+w_{0}\right) \\
& =\left(\beta^{(j)}\right)^{-1}\left(x^{(j)}+w_{0}\right)=y^{(j)} .
\end{aligned}
$$

So $\rho \circ \sigma^{-1}(w, u)=\left(y, y^{\prime}\right)$. This implies $\left(y, y^{\prime}\right) \in K$ and hence $S\left(y, y^{\prime}\right)=\left(x, x^{\prime}\right)$. On the other hand, $y \in b^{-1} \mathbb{Z}(\beta)$ because $\beta$ is an algebraic unit. So $\left(y, y^{\prime}\right)$ belongs to $\mathcal{R}_{b}$ and it is a preimage of $\left(x, x^{\prime}\right)$. This proves that $S$ is surjective on $\mathcal{R}_{b}$.

Hence $\left.S\right|_{\mathcal{R}_{b}}$ is a one-to-one mapping, and thus there exists an integer $n$ such that

$$
\left(x, x^{\prime}\right)=S^{n}\left(x, x^{\prime}\right)=\left(T_{\beta}^{n} x,\left(T_{\beta}^{n} x\right)^{\prime}\right) .
$$

Therefore $x=T_{\beta}^{n} x$; namely, the $\beta$-expansion of $x$ is purely periodic.

2.3. Example 2.1. We apply Theorem 2.1 to Pisot units of degree two (cf. [10]). the domain $K$ is illustrated by Figure 2.1. We leave the easy calculations to the readers.

Case $\beta^{2}=n \beta+1, n \geq 1$. In this case $d_{\beta}^{*}(1)=\overline{n 0}$, and $M_{\beta}$ has two states $\bar{r}_{1}=1$ and $\bar{r}_{2}=0 . \overline{0 n}=\frac{1}{\beta}$. The domain $K$ is illustrated by Figure 2.1.a. Since the line segment from $o$ to $(1,1)$ is contained in $K,\left(x, x^{\prime}\right)=(x, x)$ belongs to $K$ for any rational $0 \leq x<1$. Hence $\mathbb{Q} \cap[0,1) \subseteq \operatorname{Pur}(\beta)$, which is the result of $\mathrm{K}$. Schmidt (cf. [17]).

Case $\beta^{2}=n \beta-1, n \geq 3$. In this case $d_{\beta}^{*}=(n-1) \overline{(n-2)}, M_{\beta}$ has two states $\bar{r}_{1}=1$ and $\bar{r}_{2}=0 . \overline{(n-1)}=\frac{\beta-1}{\beta}$. The domain $K$ is illustrated by Figure 2.1.b. Now for any rational number $x \in[0,1),(x, x) \notin K$ and the $\beta$-expansion of $x$ is not purely periodic. 


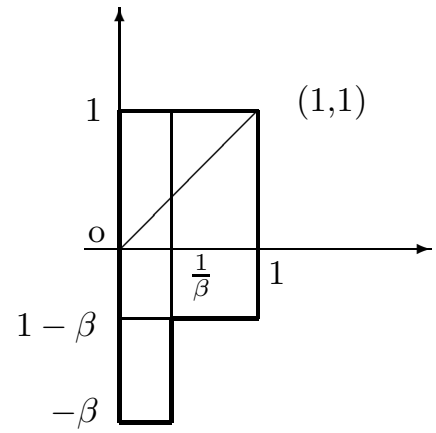

Figure 2.1.a

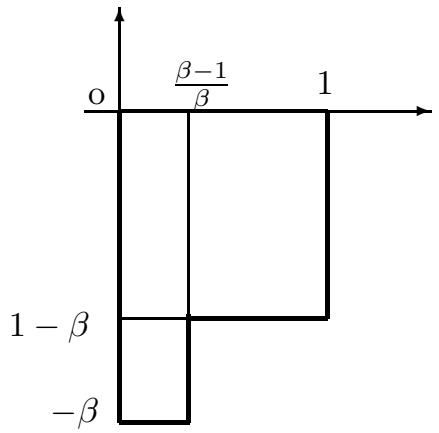

FiguRE 2.1.b

2.4. Admissible and weakly admissible. Let $\bar{\Omega}$ be the closure of $\Omega$ in the symbolic metric. Then $\bar{\Omega}$ is the set of all weakly admissible sequences. The set $\bar{\Omega}$ is compact and $\bar{K}=\rho(\bar{\Omega})$. Let $\overline{[\beta x\rfloor}$ denote the largest integer which is strictly less than $\beta x$. Replacing $\lfloor\beta x\rfloor$ in $(2.3)$ by $\overline{[\beta x\rfloor}$, we get a new operator $\bar{S}$. Similar to the above discussion, we have

Theorem 2.1'. Let $\beta>1$ be a Pisot unit. Then $x \in \operatorname{Pur}^{\prime}(\beta)$ if and only if $x \in \mathbb{Q}(\beta) \cap[0,1]$ and $\left(x, x^{\prime}\right) \in \bar{K}$.

\section{Proof of the Main Theorem}

3.1. Second realization of $(\Omega, \sigma)$. Recall that a sequence $w_{n} \ldots w_{1} w_{0}$ stops at state $i$ if there is a path form state 1 to state $i$ with label $w_{n} \ldots w_{1} w_{0}$. It is equivalent to $w_{n} \ldots w_{1} w_{0} \cdot r_{i}$ being weakly admissible, but for any $r^{\prime} \succ r$ (in lexicographical order), $w_{n} \ldots w_{1} w_{0} \cdot r^{\prime}$ is not weakly admissible. We generalize this definition to an infinite backward sequence. We say $w=\left(\ldots w_{n} \ldots w_{1} w_{0}\right)$ stops at state $i$ if $w \cdot r_{i}$ is weakly admissible but $w \cdot r^{\prime}$ is not weakly admissible for any $r^{\prime} \succ r$. We claim that

$$
X_{i}=\left\{\sum_{k=0}^{\infty} w_{k} A_{\beta}^{k} \pi\left(e_{1}\right):\left(\ldots w_{n} \ldots w_{1} w_{0}\right) \text { stops at state } i\right\} .
$$

By the definition of $X_{i}$, we have

$$
X_{i}=\overline{\pi \circ \phi\left\{\sum_{k=0}^{n} w_{k} \beta^{k}: w_{n} \ldots w_{1} w_{0} \text { stops at state } i\right\}} .
$$

Since $\pi$ and $\phi$ are linear, by (1.1) and (1.2) we see that

$$
\begin{aligned}
\pi \circ \phi\left(\sum_{k=0}^{n} w_{k} \beta^{k}\right) & =\pi\left(\sum_{k=0}^{n} w_{k} A_{\beta}^{k} \phi(1)\right) \\
& =\sum_{k=0}^{n} w_{k} A_{\beta}^{k} \pi\left(e_{1}\right) .
\end{aligned}
$$

Hence the right side of (3.1) is a subset of $X_{i}$. On the other hand, for any $x \in X_{i}$, there is a sequence of finite words $\left\{w^{[k]}\right\}_{k \geq 1}$ such that $w^{[k]}$ stops at state $i$ and the $\pi \circ \phi\left(w^{[k]}\right)$ converge to $x$. Since $\bar{\Omega}$ is compact, there is at least one limit point $w$. 
One can show that $w$ stops at state $i$ and $\sum_{k=0}^{\infty} w_{k} A_{\beta}^{k} \pi\left(e_{1}\right)=x$. This proves the other direction of (3.1).

From

$$
\left[0, \bar{r}_{i}\right]=\left\{\sum_{k=1}^{\infty} u_{k} \beta^{k}: u=\left(u_{1} u_{2} \ldots\right) \text { weakly admissible and } u \preceq r_{i}\right\}
$$

we get

$$
I_{i}=\left\{\sum_{k=1}^{\infty} u_{k} A_{\beta}^{-k} \pi^{\prime}\left(e_{1}\right): u=\left(u_{1} u_{2} \ldots\right) \text { weakly admissible and } u \preceq r_{i}\right\} .
$$

Motivated by (3.1) and (3.2), we define $\psi: \bar{\Omega} \mapsto \hat{X}$ as

$$
\psi(w, u):=-\sum_{k=0}^{\infty} w_{k} A_{\beta}^{k} \pi\left(e_{1}\right)+\sum_{k=1}^{\infty} u_{k} A_{\beta}^{-k} \pi^{\prime}\left(e_{1}\right)
$$

Proposition 3.1. $\psi(\bar{\Omega})=\hat{X}$.

Proof. Pick any $(w, u) \in \bar{\Omega}$. There is a state $i$ such that $w$ stops at state $i$ and $u \preceq r_{i}$. Therefore $\sum_{k=0}^{\infty} w_{k} A_{\beta}^{k} \pi\left(e_{1}\right) \in X_{i}$ and $\sum_{k=1}^{\infty} u_{k} A_{\beta}^{-k} \pi^{\prime}\left(e_{1}\right) \in I_{i}$. So $\psi(w, u) \in X_{i}+$ $I_{i}=\hat{X}_{i} \subset \hat{X}$. The other direction can be proved similarly.

We will show in $\S 3.4$ that $\left(\hat{X}, \hat{T}_{\beta}\right)$ is another realization of $(\Omega, \sigma)$.

3.2. Proof of the Main Theorem. Let $v=v_{1}, v_{2}, \ldots, v_{d}$ be eigenvectors of $A_{\beta}$ corresponding to eigenvalues $\beta, \beta^{(2)}, \ldots, \beta^{(d)}$ respectively. The order is arranged by (2.1). Every vector of $\mathbb{R}^{d}$ can be written in a unique way as a linear combination of $v_{1}, v_{2}, \ldots, v_{d}$. If we assume that

$$
e_{1}=v_{1}+v_{2}+\cdots+v_{d},
$$

then $v_{1}, v_{2}, \ldots, v_{d}$ are uniquely determined. Let $U=\left(v_{1}, v_{2}, \ldots, v_{d}\right)$ be a $d \times d$ matrix with entries in $\mathbb{C}$. Regarding $U$ as a linear transformation, we have

Proposition 3.2. $U(\bar{K})=\hat{X}$.

Proof. Equation (3.3) implies that $\pi^{\prime}\left(e_{1}\right)=v_{1}$ and $\pi\left(e_{1}\right)=v_{2}+\cdots+v_{d}$. Hence

$$
A_{\beta}^{k} \pi\left(e_{1}\right)=\left(\beta^{(2)}\right)^{k} v_{2}+\cdots+\left(\beta^{(d)}\right)^{k} v_{d} .
$$

Therefore,

$$
\begin{aligned}
& U(\bar{K})=U \circ \rho(\bar{\Omega}) \\
= & \left\{U\left(\sum_{k=1}^{\infty} u_{k} \beta^{-k},-\sum_{k=0}^{\infty} w_{k}\left(\beta^{(2)}\right)^{k}, \cdots,-\sum_{k=0}^{\infty} w_{k}\left(\beta^{(d)}\right)^{k}\right):(w, u) \in \bar{\Omega}\right\} \\
= & \left\{\sum_{k=1}^{\infty} u_{k} \beta^{-k} v_{1}-\sum_{k=0}^{\infty} w_{k}\left(\left(\beta^{(2)}\right)^{k} v_{2}+\cdots+\left(\beta^{(d)}\right)^{k} v_{d}\right):(w, u) \in \bar{\Omega}\right\} \\
= & \left\{\sum_{k=1}^{\infty} u_{k} A_{\beta}^{-k} \pi^{\prime}\left(e_{1}\right)-\sum_{k=0}^{\infty} w_{k} A_{\beta}^{k} \pi\left(e_{1}\right):(w, u) \in \bar{\Omega}\right\} \\
= & \psi(\bar{\Omega})=\hat{X} .
\end{aligned}
$$

Lemma 3.3. For $x \in \mathbb{Q}(\beta)$,

$$
U\left(x, x^{\prime}\right)=\phi(x)
$$


Proof. We claim that if (3.4) is true for $x$, then it is also true for $\beta x$. For

$$
\begin{aligned}
& U\left(\begin{array}{c}
\beta x \\
(\beta x)^{\prime}
\end{array}\right)=U I_{\beta}\left(\begin{array}{c}
x \\
x^{\prime}
\end{array}\right)=\left(\beta^{(1)} v_{1}, \ldots, \beta^{(d)} v_{d}\right)\left(\begin{array}{c}
x \\
x^{\prime}
\end{array}\right) \\
= & A_{\beta}\left(v_{1}, \ldots, v_{d}\right)\left(\begin{array}{c}
x \\
x^{\prime}
\end{array}\right)=A_{\beta} U\left(\begin{array}{c}
x \\
x^{\prime}
\end{array}\right)=A_{\beta} \phi(x)=\phi(\beta x) .
\end{aligned}
$$

By (3.3), we see that (3.4) is true for $x=1$. Hence (3.4) holds for $x=\beta^{k}$, $0 \leq k \leq d-1$. Notice that both $\phi$ and $x \mapsto x^{\prime}$ are linear mappings; therefore, (3.4) hold for all $x \in \mathbb{Q}(\beta)$.

Proof of the Main Theorem. Notice that the matrix $U$ is non-singular; hence,

$$
\left(x, x^{\prime}\right) \in \bar{K} \Leftrightarrow U\left(x, x^{\prime}\right) \in U \bar{K} \Leftrightarrow \phi(x) \in \hat{X} .
$$

Now our Main Theorem follows from Theorem 2.1'.

3.3. Example 3.1. Again we consider a Pisot unit of degree two.

Case $\beta^{2}=n \beta+1$. The domain $\hat{X}$ is illustrated by Figure 3.1.a. Since the line segment from $o$ to $e_{1}$ is contained in $\hat{X}$, so $\phi(x) \in \hat{X}$ holds for any rational $x$ in $[0,1)$. Hence $\mathbb{Q} \cap[0,1) \subseteq \operatorname{Pur}^{\prime}(\beta)$.

Case $\beta^{2}=n \beta-1$. The domain $\hat{X}$ is illustrated by Figure 3.1.b.

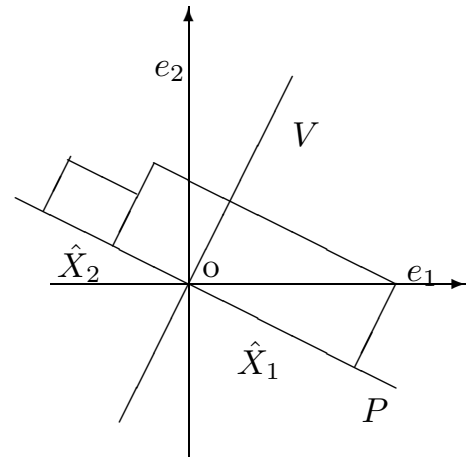

FiguRE 3.1.a

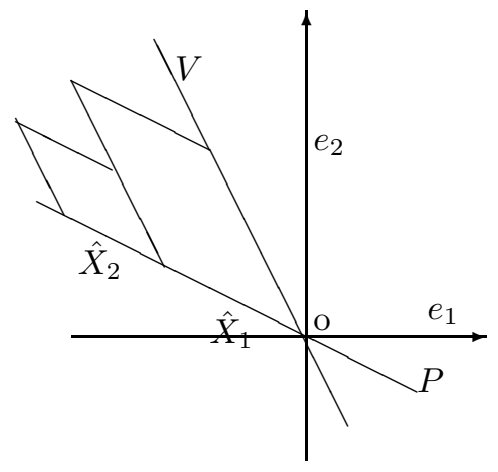

FIgURE 3.1.b

3.4. Transformation $\hat{T}_{\beta}$. Thurston [20] described the following dynamics: Let $t \in \mathbb{R}^{d}$ be a point in the tube between $P$ and the hyperplane $P+e_{1}$. Multiply $t$ by $A_{\beta}$ and subtract the largest multiple of $e_{1}$ which keeps $t$ on the same side of $P$. (Remember that $e_{1}=\phi(1)$.) Let us denote this transformation by $\hat{T}_{\beta}$. If $t=\phi(x)$ with $x \in \mathbb{Q}(\beta)$ (we say $t$ is algebraic), then it follows that

$$
\hat{T}_{\beta}(t)=\phi\left(T_{\beta} x\right) \text {. }
$$

Observe that $\hat{T}_{\beta}^{k}(t)$ always remains in a bounded region near the origin as $\hat{T}_{\beta}$ is iterated. It can never escape very far from $P$ since we always guide it back, and it can never escape very far from $V$ since $A_{\beta}$ squeezes every point toward $V$.

If $t$ starts out as an algebraic integer in $\mathbb{Q}(\beta)$, then it always remains an algebraic integer. The set of all algebraic integers forms a lattice in $\mathbb{R}^{d}$ by mapping $\phi$, so $\hat{T}_{\beta}^{k}(t)$ can only take a finite number of values. Therefore, its orbit eventually arrives back at a previous point, and from then on it repeats. If $t$ is in $\mathbb{Q}(\beta)$, then there is some integer $b$ such that $b t$ is an algebraic integer. Then we apply the above argument. This proves that every number in $\mathbb{Q}(\beta)$ has eventually a periodic $\beta$-expansion. 
From the discussion of Section 2 and Section 3, we can easily confirm the following commutative diagram, and thus $\left(\hat{X}, \hat{T}_{\beta}\right)$ is a realization of $(\bar{\Omega}, \sigma)$.

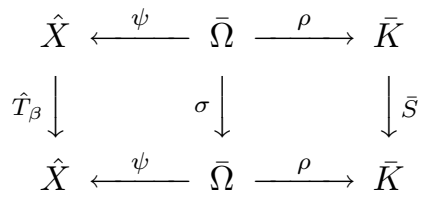

We obtain a more exact picture about the behavior of $\hat{T}_{\beta}$ : starting from an algebraic point $t$, sooner or later it will fall into $\hat{X}$. Then it moves inside $\hat{X}$, and comes back to the entering point after a finite number of steps, and from then on it repeats.

\section{SOME REMARKS}

4.1. (F)-property. We denote by $\operatorname{Fin}(\beta)$ the set of all $\alpha \in \mathbb{Z}(\beta)_{\geq 0}$ that have finite $\beta$-expansion. We say $\beta$ has the $(F)$-property (finite expansion property) provided $\mathbb{Z}(\beta)_{\geq 0} \subseteq \operatorname{Fin}(\beta)$. The $(\mathrm{F})$-property of algebraic numbers is introduced by $\mathrm{C}$. Frougny and B. Solomyak 9]. Akiyama found the connection between the (F)property and tiling.

Lemma 4.1 (Akiyama [3]). If $\beta>1$ is a Pisot unit with the (F)-property, then $0 \in P$ is an interior point of the atomic surface $X=\bigcup_{i=1}^{q} X_{i}$.

The following result of Akiyama can be derived from the Main Theorem. (Akiyama's original proof is an algebraic argument.)

Theorem A (Akiyama [2]). If $\beta>1$ is a Pisot unit with the (F)-property, then there exists a constant $c=c(\beta)>0$ such that $\mathbb{Q} \cap[0, c) \subseteq \operatorname{Pur}(\beta)$.

Proof. Let $\Delta$ be the line passing through 0 and $e_{1}$. Since $0 \in P$ is an interior point of $X, \hat{X}$ contains a small half-ball with center 0 and sitting on the positive side of $P$ (the side containing $e_{1}$ ). Hence a small part of the line $\Delta$ near 0 is contained in $\hat{X}$. We start from the origin and walk along the line $\Delta$. Let $c e_{1}$ be the first point we meet on the boundary of $\hat{X} \cap \Delta$. For a rational number $x \in[0, c), \phi(x)=x \phi(1)=x e_{1}$ belongs to the line segment from 0 to $c \phi(1)$ and thus is contained in $\hat{X}$. So by the Main Theorem, $x \in \operatorname{Pur}^{\prime}(\beta)$. Since $x$ is not an algebraic integer, it cannot be one of $r_{1}, \cdots, r_{q}$. Therefore $x \in \operatorname{Pur}(\beta)$. Moreover, this number $c$ is the best bound.

Let $\beta>1$ satisfy $\beta^{2}=n \beta+1$. Then $\beta$ has the $(\mathrm{F})$-property, and 0 is an interior point of $X$ as we see in Figure 3.1.a. In this case $c=1$. If $\beta>1$ satisfies $\beta^{2}=n \beta-1$, then $\beta$ does not have the $(\mathrm{F})$-property. From Figure 3.1.b we see that 0 sits on the boundary of $X$, and Theorem $\mathrm{A}$ is false. When $d \geq 3$, it is not so easy to get the best bound $c$ in Theorem A because of the fractal nature of atomic surfaces.

4.2. Atomic surfaces and Markov partitions. The family $\left\{X_{i}\right\}_{1 \leq i \leq q}$ has been studied extensively in many papers. It is proved that $\left\{X_{i}\right\}_{1 \leq i \leq q}$ are the invariant sets of a graph iterated function system (graph-IFS). The graph-IFS satisfies the open set condition, and the $X_{i}$ have non-empty interiors. So $\left\{X_{i}\right\}_{1 \leq i \leq q}$ forms a self-similar tiling system (cf. [16, 6, 2, 3, 4, 7, 19, 11, 8]). For detailed discussions of the tiling and dynamical properties of $\left\{X_{i}\right\}_{1 \leq i \leq q}$, we refer to [11, [8]. 
Akiyama [4] introduced the weakly finite property: $\beta$ is said to satisfy the weakly finite property if for any $x \in \mathbb{Z}(\beta)_{\geq 0}$ and any $\epsilon>0$, there exist $y, z \in \operatorname{Fin}(\beta)$ such that $x=y-z$ and $z<\epsilon$. Notice that the (F)-property implies the weakly finite property since we can always choose $z=0$. It is proved that many tiling and dynamical properties are rooted in the weakly finite property of $\beta$ (cf. [4, 11, 8]).

Notice that the domains in Figure 3.1 are essentially the Markov partitions of $A_{\beta}$ constructed by Adler and Weiss [1. It is easy to check that in Example 3.1, $\hat{X}+\mathbb{Z}^{2}$ is a lattice tiling of $\mathbb{R}^{2}$ and thus $\hat{X}$ is a two-dimensional torus. Moreover, if we regard $A_{\beta}$ as a group automorphism acting on $\hat{X}$, then it coincides with $\hat{T}_{\beta}$, and $\left\{\hat{X}_{1}, \hat{X}_{2}\right\}$ is a Markov partition for $A_{\beta}$. In general, we have the following result.

Theorem B (Ei, Ito and Rao [8]). Let $\beta$ be a Pisot unit. Then the following statements are equivalent:

(1) $\beta$ satisfies the weakly finite property;

(2) $\hat{X}+\mathbb{Z}^{d}$ is a tiling of $\mathbb{R}^{d}$, and consequently $\left\{\hat{X}_{1}, \ldots, \hat{X}_{d}\right\}$ is a Markov partition of the group automorphism $A_{\beta}$.

Akiyama [4] conjectured that any Pisot unit satisfies the weakly finite property. Some partial results are obtained in [5]. If the conjecture is true, then $\left\{\hat{X}_{1}, \ldots, \hat{X}_{q}\right\}$ always gives a Markov partition of $A_{\beta}$ and it is a generalization of the construction of Adler and Weiss [1. It is interesting that the Markov partition of $A_{\beta}$ characterizes the set of purely periodic $\beta$-expansions.

\section{ACKNOWLEDGEMENT}

The authors would like to thank S. Akiyama for many helpful discussions.

\section{REFERENCES}

[1] R. Adler and B. Weiss, Similarities of automorphisms of the torus, Memoirs of the American Mathematical Society, 98, 1970. MR0257315 (41:1966)

[2] S. Akiyama, Pisot numbers and greedy algorithm, Number Theory, Diophantine, Computational and Algebraic Aspects, Edited by K. Gyory, A. Petho and V. T. Sos, de Gruyter 1998, pp 9-21. MR 1628829 (99d:11007)

[3] S. Akiyama, Self-affine tilings and Pisot numeration system, Number Theory and Its Applications, Edited by K. Gyory and S. Kanemitsu, Kluwer, 1999, pp 7-17. MR1738803 (2001b:11094)

[4] S. Akiyama, On the boundary of self-affine tilings generated by Pisot numbers, J. Math. Soc. Japan 54:2 (2002), 283-308. MR.1883519 (2002k:11132)

[5] S. Akiyama, H. Rao and W. Steiner, A certain finiteness property of Pisot number system, J. Number Theory 107 (2004), no. 1, 135-160. MR2059954

[6] P. Arnoux and S. Ito, Pisot substitutions and Rauzy fractals, Bull. Belg. Math. Soc. 8 (2001), 181-207. MF 1838930 (2002j:37018)

[7] V. Canterini and A. Sigel, Geometric representation of primitive substitutions of Pisot type, Trans. Amer. Math. Soc. 353 (2001), 5121-5144. MR1852097 (2002f:37023)

[8] H. Ei, S. Ito and H. Rao, Atomic surfaces, tilings and coincidence II: Reducible case. preprint 2002.

[9] C. Frougny and B. Solomyak, Finite beta-expansions, Ergodic. Th. \& Dynam. Sys. 12 (1992), 713-723. MF,1200339 (94a:11123)

[10] Y. Hara and S. Ito, On real quadratic fields and periodic expansions, Tokyo J. Math. 12 (1989), 357-370. MR.1030499 (90m:11021)

[11] S. Ito and H. Rao, Atomic surfaces, tilings and coincidence I: Irreducible case. preprint 2001.

[12] S. Ito and Y. Sano, On periodic $\beta$-expansions of Pisot numbers and Rauzy fractals, Osaka J. Math. 38 (2001), 349-368. MF1833625 (2002d:11124) 
[13] S. Ito and Y. Takahashi, Markov subshifts and the realization of $\beta$-expansions, J. Math. Soc. Japan 26 (1974), 33-55. MR0346134 (49:10860)

[14] W. Parry, On the $\beta$-expansion of real numbers, Acta Math. Acad. Sci. Hung. 11 (1960), 401-416. MF 0142719 (26:288)

[15] B. Praggastis, Markov partition for hyperbolic toral automorphism, Ph.D. Thesis, Univ. of Washington, 1992.

[16] G. Rauzy, Nombres algébriques et substitutions, Bull. Soc. Math. France 110 (1982), 147-178. MR.0667748 (84h:10074)

[17] K. Schmidt, On periodic expansions of Pisot numbers and Salem numbers, Bull. London Math. Soc. 12 (1980), 269-278. MR0576976 (82c:12003)

[18] A. Siegel, Représentations géométriques, combinatoire et arithmétique des systèmes substitutifs de type Pisot, Thèsis de Doctorat, Université de la Méditérranée, 2000.

[19] V. Sirvent and Y. Wang, Self-affine tiling via substitution dynamical systems and Rauzy fractals. Pacific J. Math. 206 (2002), no. 2, 465-485. MR.1926787 (2003g:37026)

[20] W. Thurston, Groups, tilings, and finite state automata, AMS Colloquium Lecture Notes, Boulder, 1989.

Department of Information and Systems Engineering, Kanazawa University, KANAZAWA, JAPAN

E-mail address: ito@t.kanazawa-u.ac.jp

Department of Mathematics, Tsinghua University, Beijing, People's Republic of CHINA

E-mail address: hrao@math.tsinghua.edu.cn 